\title{
TRANSMISSION ULTRASONORE D'INFORMATIONS NUMÉRISÉES DANS L'EAU
}

\author{
M. ATASSI, P. BEŃARD, G. MAZE et J. RIPOCHE \\ Laboratoire d'Acoustique Ultrasonore et d'Electronique, LA.U.E. U.R.A. C.N.R.S. 1373, Université \\ le Havre, Place Robert Schuman, F-76610 Le Havre, France
}

\begin{abstract}
This paper describes an acoustic communication system that allows to transmit and to receive high-rate digital data (100 kbauds) with a directive ultrasonic beam through water. To solve the communication problem in water, the data logical levels are modulated in two opposite phases of the carrier "BPSK". To distinguish which of the two phases is the reference, the information is coded using the "OPSK" method. At the reception, the carrier is restored from the received signal to avoid the problem of Doppler effect during the transmission in water and, after an appropriate processing, the logical restored signal is then decoded using the "DEPSK" method to cancel the ambiguity of phase. To test the sensibility of system to the parasite echos, an experimental set up has been used. The emitted beam is separated in two parts, by two plane reflectors. The difference in phase and the relative amplitude are adjustable.
\end{abstract}

\section{METHODE DE TRANSMISSION}

Ce travail présente un système de transmission de données numériques par ultrasons dans l'eau à la cadence de 100 kilobauds. Les transducteurs émetteur et récepteur ont une fréquence centrale de $500 \mathrm{kHz}$ et une bande passante de $200 \mathrm{kHz}$ à $-3 \mathrm{~dB}$. Ce système est prévu pour être utilisé sur des courtes distances (une centaine de mètres). Pour être dans des conditions optimales de transmission, il faut que la bande passante du signal $\mathrm{B}_{\mathrm{S}}$ soit inférieure ou égale à celle du transducteur $\mathrm{B}_{\mathrm{t}}$. La méthode de modulation à deux états de phase "BPSK" (Binary Phase Shift Keying) avec un codage différentiel "DPSK" (Differential Phase Shift Keying) est retenue. Elle résoud le problème de l'ambiguité de l'inversion de phase. Ce choix de la méthode "BPSK-DPSK" entraine le choix d'un nombre entier de périodes de la porteuse pendant la durée d'un bit. Il est possible d'augmenter la cadence de la transmission en utilisant la méthode à 4 phases "QPSK- DPSK" [1,2] ou/et la compression des données [3]. ce qui entraîne une plus grande complexité des circuits de réception. A la réception, la porteuse est reconstituée à partir du signal reçu. Ceci permet d'éviter les problèmes dûs à l'effet Doppler pendant la transmission dans l'eau. Après plusieurs traitements (démodulation "BPSK", remise en forme), le signal binaire est restitué par un décodage différentiel "DEPSK" (Differentially Encoded PSK) pour éliminer l'ambiguité d'inversion de phase [4]. Le décodage "DEPSK" nécessite une synchronisation parfaite entre le signal binaire et l'horloge (100 kHz). La figure ( 1 ) représente le système de transmission. 


\section{RESULTATS}

Deux études expérimentales ont été effectuées:

- La première consiste à réaliser une émission directe entre deux transducteurs, afin de vérifier la concordance entre signal reçu et signal émis. La figure (II) montre les résultats de cette étude [5].

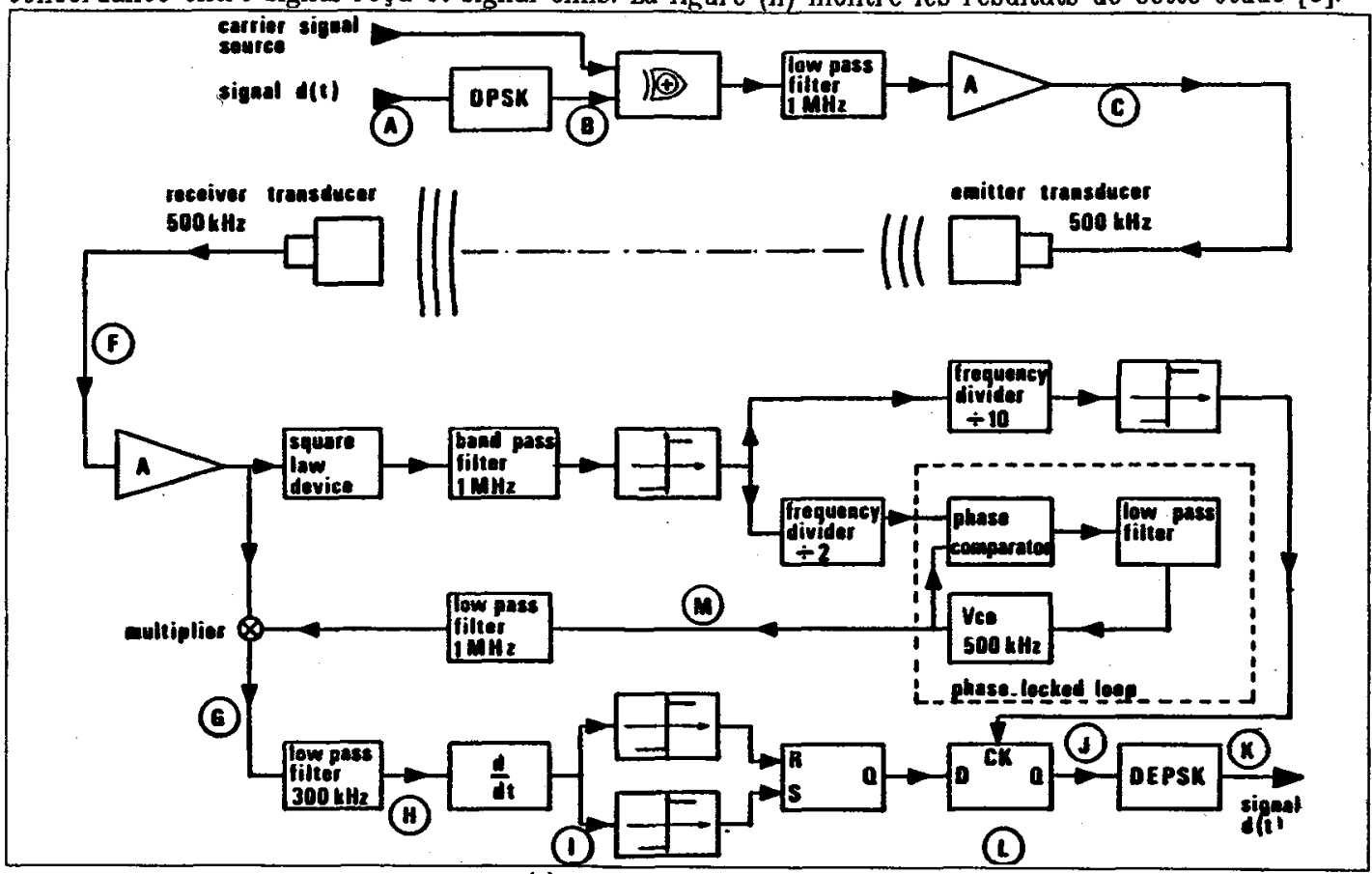

Figure (I): Schéma bloc du système

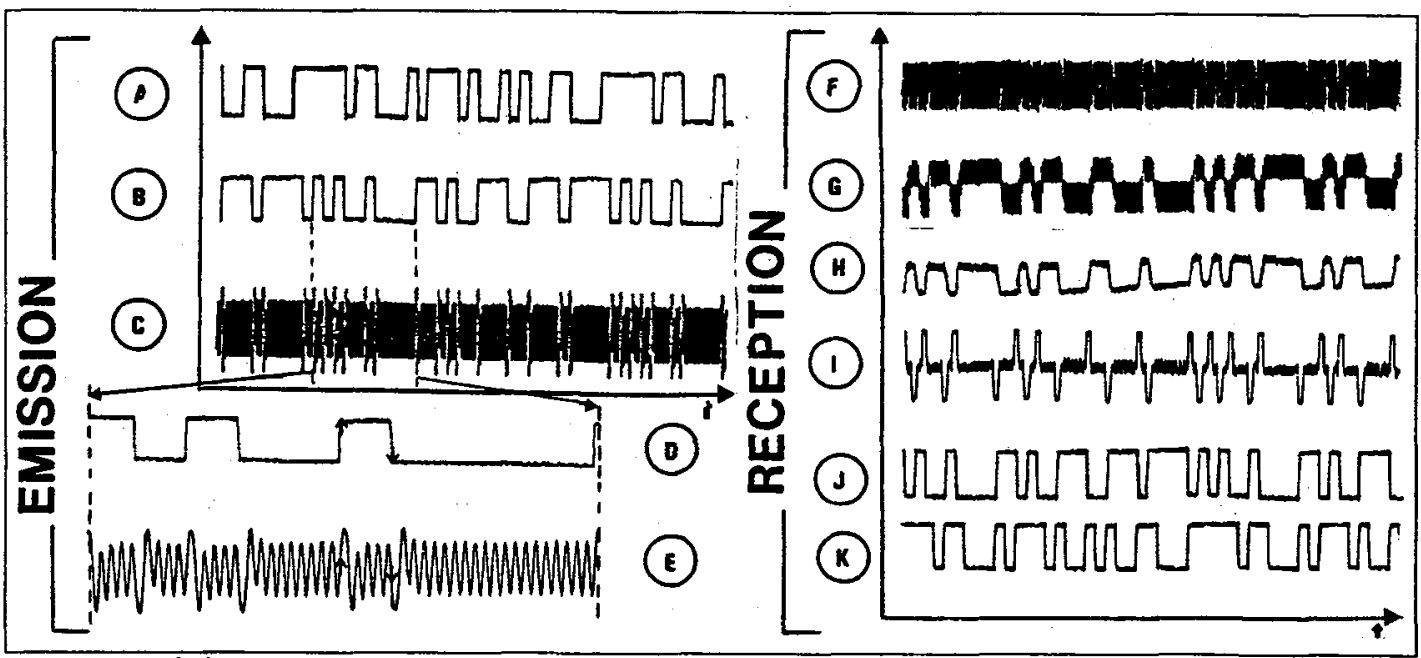

Figure (II) : Formes des signaux correspondant à différentes sorties de la figure (I).

A- Signal binaire. B- Signal binaire codé. C- Signal à transmettre.

F- Signal reçu. G- signal démodulé. H- Signal codé réstitué.

I- Signal dérivé. J-Signal codé binaire. K- Signal final.

$D$ et $E$ sont deux signaux agrandis resp. de $B$ et $C$. 
- La seconde étude est fondée sur la réflexion d'un faisceau ultrasonore sur deux surfaces planes. figure (III-1). Cette dernière étude permet de tester la sensibilité de la méthode de transmission aux échos parasites. Le faisceau émis est décomposé en deux parties à l'aide des réflecteurs plans. Leur déphasage et leur amplitude relative sont modifiables. Pour évaluer l'importance relative du signal parcourant le chemin $L_{1}$ par rapport au signal parcourant le chemin $L_{2}$, le transducteur émetteur est alimenté par un train de sinusoïde. L'amplitude relative et le déphasage sont réglés à l'aide d'un oscilloscope. Deux échos sont observables, figure (IV. F'). Dans la partie commune des échos, il peut se produire une interférence constructive ou destructive.

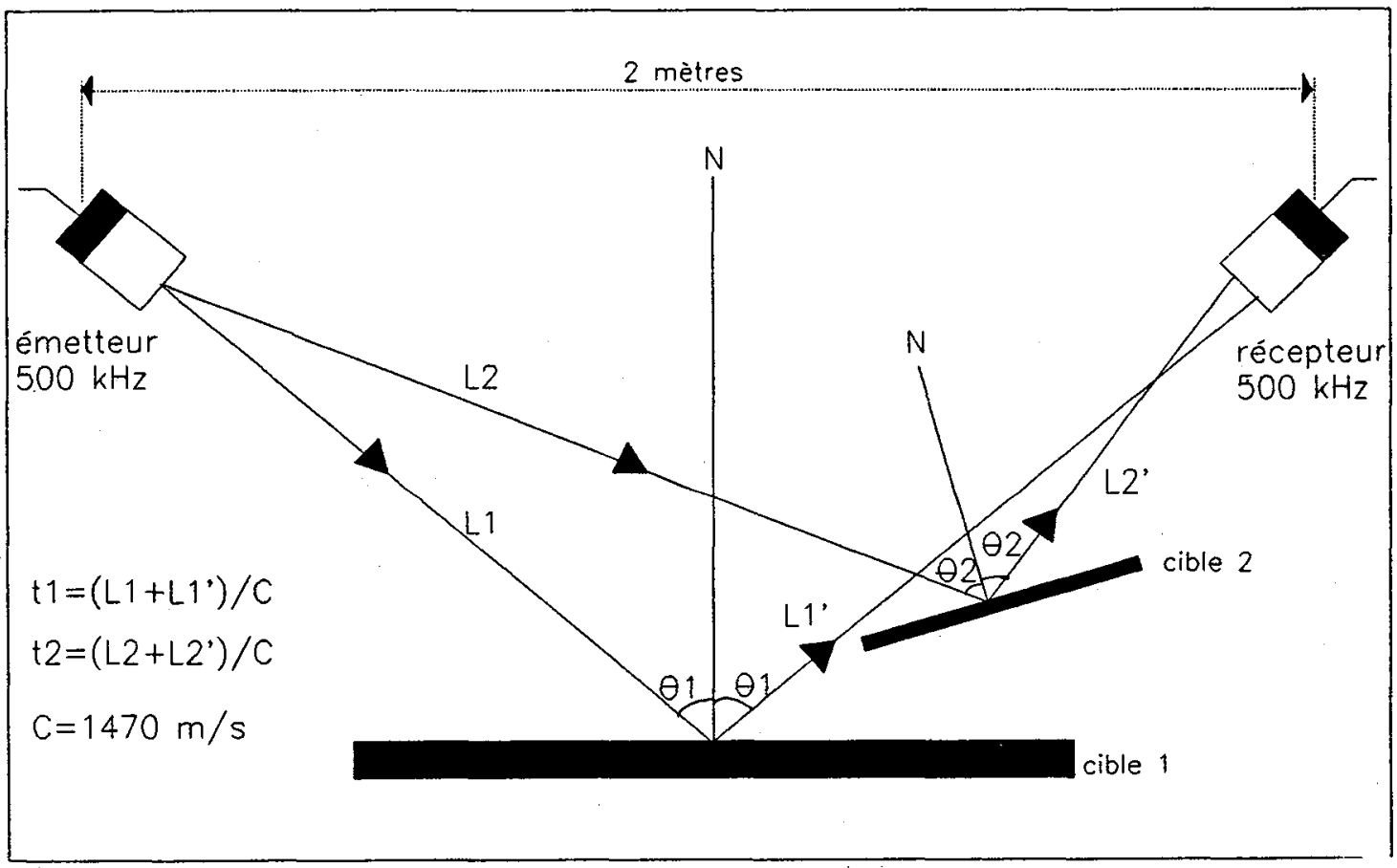

Figure (III) : Disposition des transducteurs et des réflecteurs plans

\section{CONCLUSION}

$\mathrm{Si}$ l'un des échos est beaucoup plus petit que l'autre, figure (IV.1). que l'interférence soit constructive ou destructive. il est possible de reconstituer le signal original. Si les deux échos sont d'amplitude voisine. figure (IV.2). il est encore possible de reconstituer le signal à condition d'avoir un niveau suffisant à la réception et de n'avoir ni interférence constructive ni destructive. Si les signaux sont identiques, il n'est plus possible de reconstituer le signal original. 


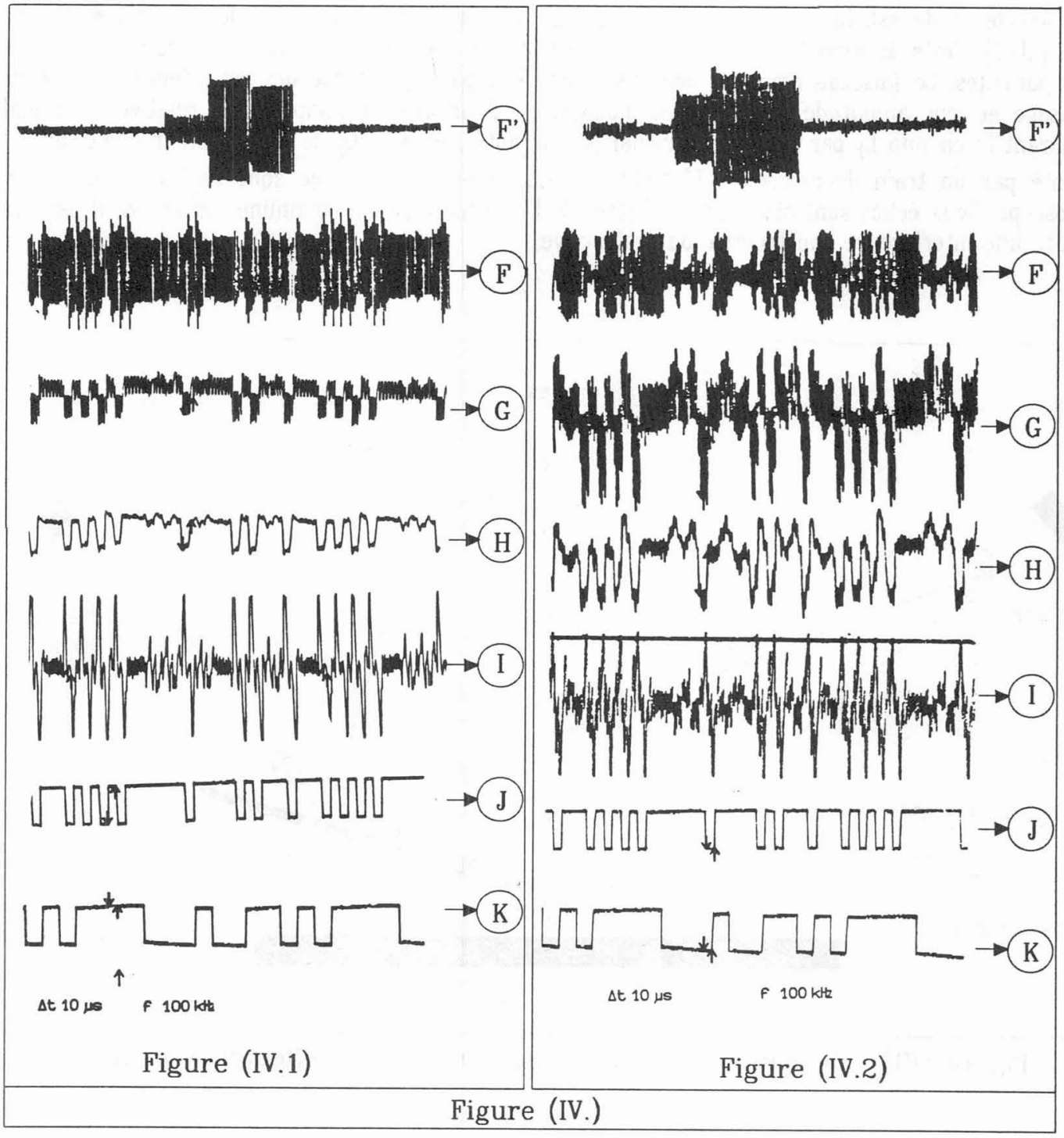

[1] B. Leduc, G. Ayela. "TIṾA a self contained image/data acoustic transmission system for underwater application". First French Conference on Acoustics. Lyon. 10-13-April 1990. Journal de Physique 51 Fasc-2 (1990) pp C2 419-C2 422.

[2] A. Glavieux. J. Labat, "Transmission d'images sur canal acoustique sousmarin". Onzième Colloque sur le Traitement du Signal et ses ApplicationsNice, 1-5 Juin1987. Proceedings pp 233 - 236.

[3] A. Maguer. "Transmission d'images sous-marines par ultrasons". Thèse de doctorat, INSA Lyon, 11. Décember 85.

[4] H. Taub. D. Schilling. "Principles of Communication Systems"(McGrawHill. Book Company 1986).

[5] M. Atassi, P. Benard. G.Maze "Underwater acoustic transmission of highrate digital data". Journal d'Acoustique, sous presse. 